\title{
Inpatient Hyperglycemia: Slide through the Scale But Cover the Bases First
}

\author{
Deepak Asudani, MD ${ }^{1}$ \\ Jorge Calles-Escandon, $\mathrm{MD}^{2}$ \\ ${ }^{1}$ Division of Medicine, Tufts University School of \\ Medicine-Baystate Medical Center, Springfield, \\ Massachusetts \\ ${ }^{2}$ Division of Endocrinology, Wake Forest Univers- \\ ity—Baptist Medical Center, Winston-Salem, North \\ Carolina
}

KEYWORDS: diabetes, inpatient hyperglycemia, hypoglycemia, insulin sliding scale.

A very compelling and growing body of evidence highlights the benefits to hospitalized patients of intensive (insulin-based) glycemic control. However, we have a tendency to attend to patients' "acute" problems during inpatient stays, and glycemic control frequently takes a backseat. As hospitalists, we frequently come across patients with diabetes admitted for various other reasons, as well as patients who develop hyperglycemia while hospitalized. During a hospital stay, it is usually not recommended that an oral hypoglycemic regimen be continued, and insulin use is necessary to more reliably control blood glucose. In this article, we emphasize the need to better manage inpatient hyperglycemia and to make a conscious effort to prescribe insulin in a more rational manner. We propose that insulin orders for an inpatient address: (1) basal insulinization, (2) meal or prandial insulin, and (3) corrective insulin. In this schema, the supplemental boluses of insulin administered to correct a blood glucose level that exceeds a set value are viewed as an adjunct to a basal/bolus insulin regimen. We also recognize the practical limitations of attaining stringent glucose targets and pinpoint those areas in need of further research.

\section{BACKGROUND}

It is not entirely clear how and when the use of the very popular insulin sliding scale as the sole approach to controlling inpatient hyperglycemia became such a widespread practice. However, the sliding scale has been passed along to subsequent generations as gospel. Despite receiving much criticism, the regular insulin sliding scale remains sacred to medical practitioners. Unfortunately, the sliding scale is very frequently the sole therapeutic tool used to control hyperglycemia, and not as a complement to a more physiologically complete (basal/bolus) insulin regimen. As attractive as the use of continuous intravenous insulin infusion is to endocrinologists, it is not frequently used outside intensive care units for many reasons. Where there is apparent agreement is in the need to improve inpatient management of hyperglycemia.

\section{THE PROBLEM: HYPERGLYCEMIC INPATIENT}

Hyperglycemia is defined as a fasting glucose level greater than $126 \mathrm{mg} / \mathrm{dL}$ or 2 or more random blood glucose levels greater than $200 \mathrm{mg} / \mathrm{dL}^{1}{ }^{1}$ Not infrequently, patients admitted to our ward have a history of diabetes; however, a good proportion of admitted 
patients have no such history. In a retrospective analysis of more than 2000 consecutive hospital admissions, hyperglycemia was found in as many as $38 \%$ of the patients in whom blood glucose was measured and documented in the chart, about a third of which did not previously carry the diagnosis of diabetes. Hyperglycemia in this specific setting, dubbed "stress hyperglycemia," ${ }^{1}$ is quite frequently found in hospitalized patients and has been shown to increase the risk of death, congestive heart failure, and cardiogenic shock after myocardial infarction. ${ }^{2}$ Acute insulin resistance is also seen frequently in an acutely ill patient and is attributed to the release and metabolic actions of counterregulatory hormones and cytokine excess. ${ }^{3}$ Patients often require increased amounts of insulin to maintain glucose at an acceptable level. Iatrogenic hyperglycemia may occur as a consequence of glucocorticoids or excessive infusion of dextrose. In critically ill patients, vasopressors may also be associated with iatrogenic hyperglycemia. Inpatient hyperglycemia is associated with nosocomial infections, increased mortality, increased length of stay, and poor overall outcome. ${ }^{4}$ Of interest is that stress hyperglycemia was associated with more adverse outcome than was hyperglycemia in a patient with known diabetes. ${ }^{1,2}$ We are not sure if this phenomenon of stress hyperglycemia is pathogenic or serves as a marker of disease severity.

\section{Is Hyperglycemia Really a Problem?}

Compelling evidence that control of hyperglycemia improves the outcomes of patients undergoing cardiothoracic surgery was provided by the Portland trial. Although this study was not randomized and its glycemia targets were not well defined, it demonstrated that better control of blood glucose levels drastically reduces the incidence of chest wall infections and the need for transfusions and significantly shortens hospital length of stay (LOS).$^{5}$

The results of the Diabetes Mellitus InsulinGlucose in Acute Myocardial Infarction (DIGAMI) study showed that hyperglycemic patients with acute myocardial infarction had improved outcomes when intravenous administration of insulin was used to aggressively control glycemia. ${ }^{6}$ Van den Berghe et al. found significantly lower mortality and morbidity rates in surgical intensive care unit patients in whom aggressive glycemic control was attained with continuous intravenous insulin infusion. The study also identified reduced requirement of antibiotics, red cell transfusions, dialysis, and ventilatory support with aggressive glycemic control. ${ }^{7}$ It was also shown that there was significantly reduced morbidity in all patients in the medical ICU receiving intensive insulin therapy. ${ }^{8}$ Another meta-analysis found that insulin therapy initiated in hospitalized critically ill patients in different clinical settings had a beneficial effect on short-term mortality. ${ }^{9}$ Krinsley observed hyperglycemia to be associated with adverse outcomes in acutely ill adult patients and that its treatment has been shown to improve mortality and morbidity in a variety of settings. ${ }^{10}$ In their study of adults with diabetes, Golden et al. identified hyperglycemia as an independent risk factor for surgical infection of diabetic patients undergoing cardiac surgery. ${ }^{11} \mathrm{~A}$ meta-analysis by Capes et al. showed a 3-fold higher risk of poor functional recovery in nondiabetic hyperglycemic patients compared to that of nondiabetic euglycemic patients. ${ }^{2}$ A recent retrospective analysis found that patients with hyperglycemia treated for acute exacerbation of chronic obstructive pulmonary disease had poor outcomes. ${ }^{12}$

It is possible to give an account and references of only a limited number of such studies. The prevailing message conveyed in all these studies is that patients with poorly managed hyperglycemia have a poor overall outcome. Hence, the need to better manage inpatient hyperglycemia cannot be overemphasized. ${ }^{13}$

After an extensive search, we could not find well-designed prospective randomized studies of patients who are not acutely ill or are outside the perisurgical period. However, the DIGAMI, Van den Berghe, and Portland trials generated a powerful and large momentum that has created interest in establishing protocols for keeping the blood glucose of patients in most medical and surgical critical care units in the suggested range. ${ }^{5-7,13}$ Moreover, extrapolation of the data to noncritical and nonsurgical patients made possible a consensus conference organized by the American Association of Clinical Endocrinologists (AACE) that garnered support from many other medical associations. The position paper published by the AACE calls for tighter glycemic control in hospitalized patients. The AACE recommends that blood glucose concentrations for intensive care unit patients be maintained below $110 \mathrm{mg} / \mathrm{dL}$. In noncritically ill patients, the preprandial glucose level should not exceed $110 \mathrm{mg} / \mathrm{dL}$, and maximum glucose should not exceed $180 \mathrm{mg} / \mathrm{dL}^{.}{ }^{14}$ The American Diabetes Association (ADA) does not recommend any target 
glucose values for noncritical patients but does believe there is a need to have better inpatient hyperglycemic management. Some authorities believe that until the amount of scientific data increases, it is prudent to stay within the ADA-recommended ambulatory guidelines for a preprandial plasma glucose level of $90-130 \mathrm{mg} / \mathrm{dL}^{15}$ and a postprandial blood glucose level not to exceed $180 \mathrm{mg} / \mathrm{dL}$.

Additionally, due attention must be paid to hypoglycemia secondary to aggressive glycemic control.

Because of the absence of evidence-based information, it is not surprising that opinions conflict about the optimal level of blood glucose for an inpatient. We believe that in the absence of definitive evidence, it is prudent to adhere to the targets recommended by these associations.

\section{A SOLUTION: WHAT TO DO AND HOW TO DO IT}

Ideally, a system should be established to attain euglycemia without the attendant risk of hypoglycemia. The Joint Commission on Accreditation of Healthcare Organizations recently showed recognition of this need this by collaborating with the American Diabetes Association to establish a program to certify inpatient diabetes care center programs that meet national standards. The program must be carried out in all inpatient settings and should include the following elements ${ }^{16}$ :

- Specific staff education requirements;

- Written blood glucose-monitoring protocols;

- Plans for the treatment of hypoglycemia and hyperglycemia;

- Collection of data on the incidence of hypoglycemia;

- Education of patients on self-managing their diabetes; and

- An identified program champion or program champion team.

The Joint Commission's Advanced Inpatient Diabetes Certification Program is based on the ADA guidelines; the scope of this manuscript does not cover all the elements required to receive certification. ${ }^{16}$ In the rest of the article, we focus on the basic principles of the use of insulin to control hyperglycemia in the hospital setting.

The normal system that regulates glycemia encompasses a very complex system of hormonal and metabolic regulators. At the core of this system, insulin is the key regulator. Therapeutic insulin is therefore the best resource available for controlling hyperglycemia in the hospital setting.
Of the other currently available therapies, none offers the power and rapidity that insulin has to control blood glucose level. The biguanides are usually contraindicated in the hospital setting because most patients with hyperglycemia and/or diabetes are acutely ill and hence at risk of lactic acidosis. Furthermore, in a large number of these patients radio-contrast agents are used; hence, transient renal failure is common, posing yet another risk factor for lactic acidosis. The thiazolidinediones (TZDs) are slow to act and not as powerful in controlling acute hyperglycemia and thus are not the optimal tool available when the metabolic situation changes drastically as occurs in hospitalized patients. Precaution needs to be taken when using TZD to treat patients who have congestive heart failure or hepatic insufficiency. The action of the sulfonylureas (SUs) imparts a high risk of hypoglycemia and/or poor insulinemic response during stress to patients being treated with them; therefore, it is usually recommended that patients in a hospital setting not be treated with SUs, except for selected very stable patients. The new emerging therapies (incretin mimetics, dipeptidyl peptidaseIV inhibitors, amylin) have never been tested in the hospital setting, and hence no recommendation can be made at this stage. Thus, we believe that the main tool available for treating the hospitalized patient with hyperglycemia is insulin coupled with proper nutrition and a system of information to monitor therapeutic progress, which allows for proper and timely adjustments as well as for treatment of hypoglycemia.

Within this setting a conceptual frame for insulin administration has been proposed. Exogenous insulin needs to be provided to mimic as closely as possible the physiological pattern of endogenous insulin secretion. The latter is broadly thought to be composed of 2 secretory components: a basal component and a prandial, or alimentary, component. The basal component of insulin secretion represents the rate of insulin produced independent of meal ingestion, which is mainly governed by the prevailing concentrations of arterial blood glucose and other hormonal and metabolic regulators. Prandial insulin is the increase in insulin secretion that occurs after eating, which occurs as a complex pattern of pulses. Roughly, prandial insulin secretion is mainly determined by the quantity and composition of the meal ingested, especially the quantity of carbohydrate.

Thus, the insulin dose that an inpatient re- 
quires may be thought as consisting of "basal" and "nutritional" insulin requirements. To these 2 components we also add a third component: a "correctional" insulin component.

The basal insulin requirement of a given patient can be estimated by taking into consideration the type of diabetes and body weight. The nutritional insulin requirement refers to the insulin required to cover nutritional intake, which in a hospital setting may correspond to regular meals, intravenous dextrose, nutritional supplements, enteral feedings, or parenteral nutrition. Because our estimates are not very accurate, corrective insulin is required to correct elevated concentrations of plasma glucose (usually measured with finger sticks) A scale or table of corrective insulin can be constructed on the basis of type of diabetes, body weight, and/or total amount of daily doses of basal and nutritional insulin. Obviously, many will think of corrective insulin as a sliding scale. It is import to remember that this scale is complementary to prescribed basal and nutritional insulin doses and not a substitute for them.

How can insulin be prescribed in the hospital to cover the 3 facets of insulin (basal, alimentary, and corrective)? The following is a pragmatic approach that we found useful and uses the above considerations as an underpinning. We will first consider the general medicine or surgical ward and then the intensive care setting.

\section{General Medical and Surgical Wards Basal insulin}

The activity of the ideal insulin preparation for this task should not show any peak, instead should remain in a steady state for 24 hours. Currently, three insulin preparations can be considered for this purpose. Glargine insulin is an analogue of insulin that has a stronger capacity to form and maintain hexamers of insulin and its rate of absorption from the subcutaneous depot, which allows for quasisteady-state action for 24 hours. However, variability is sometime noted clinically in the length of duration and the absence or presence of a peak. Neutralized protamine insulin (NPH) is a mixture of protamine and human insulin in which the complexing of the 2 proteins retards absorption of insulin. The action profile of NPH insulin definitively displays a peak (between 6 and 10 hours after injection); however, the timing of this peak varies from patient to patient and (in the same patient) from day to day. There is also variability in the

\section{Key Points in Inpatient Hyperglycemia Management}

- Management of inpatient hyperglycemia is very crucial and should be given adequate attention.

- A complete insulin regimen for management of inpatient hyperglycemia consists of basal, nutritional, and correctional insulin.

- Correctional insulin or the so-called sliding scale should not be used in a stand-alone way to correct hyperglycemia.

- The use of steroids and the type of steroid used have implications on the type of insulin used.

- Oral hypoglycemics are frequently discontinued for inpatients.

- Hyperglycemic correction should not be overzealous, and hypoglycemia needs to be addressed promptly.

- Adequate hyperglycemia control has significant implications for patient morbidity and mortality.

- Insulin therapy, in both critical and noncritical settings should be frequently monitored, preferably with personnel devoted to managing inpatient glycemic control.

- Insulin dosing may be empirically initiated (see article) and needs to be frequently adjusted.

FIGURE 1. Key points in inpatient hyperglycemia management.

widely quoted duration of action (12-18 hours). Despite these shortcomings, NPH has been used for several decades and has widespread acceptance among physicians, especially because it costs less than glargine insulin. Detemir insulin is a new analogue of insulin. The insulin molecule has been complexed with a fatty acid. This modification protracts absorption from the subcutaneous depot and also within the blood compartment because the acylated insulin binds to albumin, which then acts as a reservoir. There is very little experience with detemir in clinical scenarios and none in the hospital setting.

Our preferred basal insulin, given current knowledge and experience, is glargine, except in those special cases in which insulin that has a shorter 
action is needed (ie, patients with tube feeds, use of steroids), listed below in the Special Considerations section.

\section{Alimentary or nutritional or prandial or bolus insulin}

The ideal insulin to cover the prandial period should have rapid onset and rapid dissipation of activity. Two types of insulin preparation have these characteristics. The insulin traditionally used in this setting is regular human insulin. Unfortunately, the onset of action of this preparation is not as rapid (20-30 minutes), forcing it to be prescribed as a preprandial insulin. In a hospital setting, where the dynamics of meal serving and NPO periods are highly unpredictable, this creates a serious risk of hypoglycemia when preprandial insulin is the choice. The activity of regular human insulin lasts 4-6 hours, and thus there is also the risk of stacking multiple prandial doses and hence an increase in the risk of hypoglycemia. So, although this insulin has been used for many decades, it has been rapidly replaced by the new analogues of insulin (lispro, aspart, and glulisine), which have a much faster onset of activity (within 15 minutes of injection) because of its rapid absorption from the subcutaneous depot. Moreover, the dissipation of insulin action of these preparations is faster (3-4 hours). The minor decrease in glycemic power at equivalent doses compared to preprandial administration can usually be easily overcome with a minimal increase in dosage. It is becoming widely accepted because it allows for flexibility in the timing of administration (which can be made contingent on meal ingestion) and also in dosing because it can be better tailored to the amount of food consumed. Overall, our preference is for the use of analogues in the hospital setting. The only drawback is cost.

\section{Corrective insulin}

The type of insulin used for the correction of glycemia that exceeds the target follows from the same considerations as those used for the alimentary or prandial insulin. For simplicity, the same type of insulin chosen for alimentary insulin should be the one selected for corrective insulin.

\section{How to dose the insulin?}

Once the type of insulin preparations has been chosen, dosing is the next task. The hospitalist should remember that the initial prescription or dosage of insulin will need to be reevaluated daily to allow for glycemia management and to avoid hypoglycemia. As in many other fields of medicine, a single approach does not fit all scenarios. The following is a list of scenarios commonly encountered in our inpatient population. There is no definitive way to suggest how successfully they are managed as outpatients. It may be reasonable to assume glycemic control with $\mathrm{HbAlC}$ of less than $7 \%$ as being highly successful, HbAlc between $7.1 \%$ and $8.5 \%$ as being moderately successful, and HbAlC greater than $8.5 \%$ as being unsuccessful. These HbAlC levels help to guide us through decision making and have been very helpful in our practice. Recognition of hyperglycemia either on admission or during an in-hospital stay warrants consideration of insulin-based management. For several reasons, HbAlc should be tested in patients who are found to have hyperglycemia in the hospital. Elevated glycated hemoglobin enables the recognition of previously undiagnosed diabetes and helps in the identification of patients with poorly controlled diabetes; hence, the hospital stay can be an opportunity to change treatment approaches or emphasize compliance. Likewise, in a hyperglycemic patient with normal $\mathrm{HbAlC}$, it should be considered whether stress hyperglycemia has developed.

1. Patient is using a highly successful regimen with oral agents. Recommendation: continue using oral agents if no contraindications exist, the patient is unlikely to receive contrast dye tests, and admission is for a minor indication requiring a short inpatient stay. Oral agents may need to be stopped if poor glycemic control after hospitalization or any contraindication is identified.

2. Patient is using a regimen of insulin that has been very successful. Recommendation: follow the same regimen and add to it the table for correction insulin. 3. Patient is using a regimen of insulin that is moderately successful. Recommendation: keep the same regimen but increase doses and add the correction insulin table.

4. Patient is using an unsuccessful oral regimen. Recommendation: discontinue oral agents and start basal, alimentary, and corrective insulin

5. Patient is using a very unsuccessful regimen of insulin. Recommendation: reevaluate and prescribe a basal, alimentary, and correction insulin regimen.

6. Patient is recently or newly diagnosed with diabetes. Recommendation: while in the hospital use a basal, alimentary, and corrective insulin regimen.

7. Patient has type 1 diabetes. Recommendation: 
prescribe full insulin coverage with basal, nutritional, and correctional insulin.

8. Patient is receiving an IV drip of insulin and is no longer critical and tolerating po intake. Recommendation: overlap IV drip with subcutaneous insulin for at least 4 hours and then continue subcutaneous insulin.

Empirical calculation of basal insulin. Once you have decided which insulin to use as basal insulin, the following may be used to calculate empirical doses. Suggested insulin types for basal include glargine and NPH insulin.

For scenarios $4,5,6$, and 7 we use a simple formula to estimate the basal insulin requirements. Longer-acting insulin requirements may be calculated as:

For type 2 diabetes: 0.4 units $/ \mathrm{kg} /$ day of basal insulin. For type 1 diabetes: 0.2 units $/ \mathrm{kg} /$ day of basal insulin.

The adjustments should be made every 48 hours 2-5 units at a time or $10 \%$ of the dose.

For a regimen based on glargine insulin: full dose is administered daily.

For NPH: two thirds is given AM and one third PM.

For scenario 8: basal insulin is estimated as total dose of insulin drip per hour for the last 6 hours $\times 0.8$ $\times 24$. We recommend an overlap of IV drip and SQ insulin of at least 4 hours.

Empirical calculation of alimentary or nutritional insulin. Once the type of insulin has been chosen, the insulin doses may be calculated empirically. Suggested insulin choices include lispro, aspart, and glusiline insulin.

As a convenient tool, the total daily alimentary or nutritional insulin requirement is nearly equal to total daily basal insulin. This dose estimation may then be divided into various premeal doses on the basis of the carbohydrate content of the meal. Provision of 1 unit of short-acting insulin should be made for every 15 units of carbohydrate intake. The following rough estimation may be used to calculate the premeal alimentary insulin dose.

For type 2 diabetes: Empirically, 0.1, 0.15, and 0.15 units of short-acting insulin $/ \mathrm{kg}$ for breakfast, lunch, and dinner, respectively.

For type 1 diabetes: Empirically, we suggest 0.05-0.1 units of rapid-acting insulin $/ \mathrm{kg}$, to be administered before meals.

The premeal dose requirement of an individual patient may be significantly different. If patient is
NPO, then alimentary insulin is not prescribed; specific doses need to be suspended if patient is made NPO and resumed when PO is restored.

Total daily dose of insulin may vary according to body weight, endogenous insulin secretory capacity, and degree of insulin resistance. Variation tends to be greater for those with type 2 diabetes.

We encourage administration of alimentary insulin at or immediately after meal ingestion. This implies a system of alert for patients to let nurses know when they have finished eating.

Empirical calculation of correctional insulin (sliding scale). Although every institution relies on its own guidelines, we reproduce here the correction table we use at Wake Forest University-Baptist Medical Center (WFUBMC), which was generated based on type of diabetes and body weight:

In our inpatient practice this correction table has been quite helpful. Bear in mind that there are several correctional insulin dose algorithms, and the one most suitable to local needs should be adopted.

Once the insulin to be used for correctional insulin has been chosen, the algorithm shown in Figure 2 may be used.

To ensure clarity about the prescribed regimen, we encourage the use of preformatted templates or (preferably) computerized orders. Figure 3 shows an example, an order template that we use at WFUBMC.

\section{Intensive Medical and Surgical Care Unit}

Continuous intravenous insulin infusion (IV insulin drip) is the most suitable way to administer insulin to critically ill patients. For continuous intravenous insulin infusion, regular insulin is most commonly used and in fact is the only type of insulin studied in prospective randomized trials. This requires adequate staffing, frequent monitoring, and frequent dose adjustments. Such stringent glycemic control is appropriate for patients in critical care units. The American College of Endocrinology recommends using intravenous insulin therapy in the subset of inpatients who have diabetic ketoacidosis, are before major surgical procedures, are undergoing fasting for more than 12 hours and have type 1 diabetes, are critically ill, are undergoing labor and delivery, are being treated for myocardial infarction, have just had organ transplantation, are being maintained on total parenteral nutrition, or have other illnesses requiring prompt glucose control. ${ }^{14}$

The use of continuous intravenous insulin infusion on a regular basis on all medical floors is not 
CORRECTION INSULIN ALGORITHM - TYPE 2 DIABETES/STRESS HYPERGLYCEMIA

\begin{tabular}{|c|c|c|c|c|c|c|c|}
\hline \multirow{2}{*}{$\begin{array}{c}\text { FSBS } \\
\text { (mg/dL) } \\
\nabla\end{array}$} & \multirow[t]{2}{*}{ Wt. } & $\underset{<70 \mathrm{~kg}}{\mathrm{~A}}$ & $\begin{array}{c}\text { B } \\
71-100 \mathrm{~kg}\end{array}$ & $\underset{101-130 \mathrm{~kg}}{\mathrm{C}}$ & $\underset{131-170 \mathrm{~kg}}{\mathrm{D}}$ & $\begin{array}{c}E \\
>170 \mathrm{~kg}\end{array}$ & \multirow[t]{2}{*}{ CUSTOM } \\
\hline & & \multicolumn{5}{|c|}{ UNITS OF INSULIN } & \\
\hline $150-200$ & & 1 & 2 & 3 & 4 & 5 & \\
\hline $201-250$ & & 3 & 5 & 6 & 9 & 10 & \\
\hline $251-300$ & & 5 & 7 & 10 & 13 & 15 & \\
\hline $301-350$ & & 6 & 10 & 13 & 17 & 20 & \\
\hline $351-400$ & & 8 & 12 & 15 & 20 & 25 & \\
\hline$>400$ & & 9 & 15 & 20 & 25 & 30 & \\
\hline
\end{tabular}

CORRECTION INSULIN ALGORITHM - TYPE 1 DIABETES

\begin{tabular}{|c|c|c|c|c|c|c|c|}
\hline \multirow{2}{*}{$\begin{array}{c}\text { FSBS } \\
\text { (mg/dL) } \\
\nabla\end{array}$} & \multirow[t]{2}{*}{ Wt. } & $\underset{<70 ~ k g}{F}$ & $\underset{71-100 ~ k g}{G}$ & $\begin{array}{c}\mathrm{H} \\
101-130 \mathrm{~kg}\end{array}$ & $\stackrel{\mathrm{I}}{131-170 \mathrm{~kg}}$ & $\underset{>170 \mathrm{~kg}}{\mathrm{~J}}$ & \multirow[t]{2}{*}{ custom } \\
\hline & & \multicolumn{5}{|c|}{ UNITS OF INSULIN } & \\
\hline $150-200$ & & 1 & 1 & 2 & 2 & 3 & \\
\hline $201-250$ & & 2 & 3 & 3 & 5 & 5 & \\
\hline $251-300$ & & 3 & 4 & 5 & 7 & 8 & \\
\hline $301-350$ & & 3 & 5 & 7 & 8 & 10 & \\
\hline $351-400$ & & 4 & 6 & 9 & 10 & 13 & \\
\hline$>400$ & & 5 & 8 & 10 & 12 & 15 & \\
\hline
\end{tabular}

FIGURE 2. Corrective insulin dose algorithm. Multiply by 0.055 to convert $\mathrm{mg} / \mathrm{dL}$ to $\mathrm{mmol} / \mathrm{L}$.

routinely recommended because there is not adequate scientific data to support its use from both clinical and financial perspectives. There are various protocols for attaining recommended levels, and every institution must adopt or develop a protocol that both suits its needs and is feasible. Again, aggressive glycemic control using intravenous insulin requires a well-monitored setup and is ideal for intensive care units.

\section{Management of hypoglycemia}

The therapeutic window between insulin effectiveness and insulin-associated hypoglycemia is very narrow; hence, proper management of blood glucose needs to be embedded within a system in which every member of the team taking care of a patient with diabetes has appropriate knowledge of the task at hand. Of equal importance is the devel- opment of a protocol to treat hypoglycemia minimizing the overzealous treatment that leads to severe hyperglycemia. This protocol also assumes that all oral hypoglycemic agents have been discontinued.

\section{Hypoglycemia protocol (FSBS $<70 \mathrm{mg} / \mathrm{dL}$ )}

Patient conscious and able to eat (select one):

- Provide patient with $15 \mathrm{~g}$ of carbohydrate (120 cc of fruit juice or $180 \mathrm{cc}$ of regular soda or $240 \mathrm{cc}$ of skim milk or 3 glucose tablets).

- Recheck fingerstick blood sugar (FSBS) in 15 minutes.

- Repeat above if FSBS still $<70 \mathrm{mg} / \mathrm{dL}$; continue cycle until FSBS is $>70 \mathrm{mg} / \mathrm{dL}$.

- Once FSBS is $>70 \mathrm{mg} / \mathrm{dL}$, recheck FSBS in 1 hour; if it is $<70 \mathrm{mg} / \mathrm{dL}$, repeat above cycle and call HO. 
NORTH CAROLINA BAPTIST HOSPITAL

\section{PHYSICIAN ORDER FORM}

Addressograph

PHYSICIANS: All orders should be written

generically and using the metric system; include

physician's signature, PRINTED name, ID number,

beeper number, and date/time. A generically and

therapeutically alternative drug as approved by the

P \& T Committee may be dispensed unless the order

is specifically designated "Dispense as Written."

Form approved by Medical Record Informatics Technology Committee 03/06

FAX:

TITLE: Glycemia Control Management Protocol

\begin{tabular}{|l|l|}
\hline DATE & \\
\hline TIME & (PLEASE CIRCLE OR CHECK APPROPRIATE ORDERS AND FILL IN BLANKS AS NEEDED) \\
\hline
\end{tabular}

DIAGNOSIS:

ALLERGIES:

Correction Insulin Algorithm (administer subcutaneously) ${ }^{* *}$ Select same insulin type as meal insulin** Give correction insulin dose, if needed, at the same time as scheduled insulin dose. Correction dose should be given, if indicated, based on FSBS, even when there are NO scheduled insulin doses.

$\square$ Type 2 diabetes/stress hyperglycemia

$\square$ Type 1 diabetes

Body weight $\mathrm{kg}$

Select Correction Insulin Algorithm based on body weight and type of diabetes. When steroids are used, select scale based on type of diabetes and weight, then choose the next higher scale.

Must circle appropriate letter for patient or fill in custom boxes

$\square$ Lispro $\square$ Aspart $\square$ Regular

\begin{tabular}{|c|c|c|c|c|c|c|}
\hline \multicolumn{7}{|c|}{ CORRECTION INSULIN ALGORITHM } \\
\hline \multicolumn{7}{|c|}{ TYPE 2 DIABETES/STRESS HYPERGLYCEMIA (dosed in units) } \\
\hline FSBS $\boldsymbol{\nabla}$ & $\begin{array}{c}A \\
<70 \mathrm{~kg}\end{array}$ & $\begin{array}{c}\text { B } \\
71-100 \mathrm{~kg}\end{array}$ & $\begin{array}{c}C \\
101-130 \mathrm{~kg}\end{array}$ & $\begin{array}{c}D \\
131-170 \mathrm{~kg}\end{array}$ & $\begin{array}{c}E \\
>170 \mathrm{~kg}\end{array}$ & CUSTOM \\
\hline $150-200$ & 1 & 2 & 3 & 4 & 5 & \\
\hline $201-250$ & 3 & 5 & 6 & 9 & 10 & \\
\hline $251-300$ & 5 & 7 & 10 & 13 & 15 & \\
\hline $301-350$ & 6 & 10 & 13 & 17 & 20 & \\
\hline $351-400$ & 8 & 12 & 15 & 20 & 25 & \\
\hline$>400$ & 9 & 15 & 20 & 25 & 30 & \\
\hline \multicolumn{7}{|c|}{ TYPE 1 DIABETES (dosed in units) } \\
\hline FSBS $\nabla$ & $\begin{array}{c}\mathrm{F} \\
<70 \mathrm{~kg}\end{array}$ & $\begin{array}{c}\mathbf{G} \\
71-100 \mathrm{~kg}\end{array}$ & $\begin{array}{c}\mathrm{H} \\
101-130 \mathrm{~kg}\end{array}$ & $\begin{array}{c}\mathrm{I} \\
131-170 \mathrm{~kg}\end{array}$ & $\underset{>170 \mathrm{~kg}}{\mathrm{~J}}$ & CUSTOM \\
\hline $150-200$ & 1 & 1 & 2 & 2 & 3 & \\
\hline $201-250$ & 2 & 3 & 3 & 5 & 5 & \\
\hline $251-300$ & 3 & 4 & 5 & 7 & 8 & \\
\hline $301-350$ & 3 & 5 & 7 & 8 & 10 & \\
\hline $351-400$ & 4 & 6 & 9 & 10 & 13 & \\
\hline$>400$ & 5 & 8 & 10 & 12 & 15 & \\
\hline
\end{tabular}

Physician SIGNATURE:

Physician NAME (print):

Beeper \#:

Unit Secretary SIGNATURE:

TIME Sent to Pharmacy:

RN SIGNATURE: 
Patient NPO or unconscious and IV access available

- Administer $15 \mathrm{~mL}$ of $50 \%$ dextrose IV (mix in $25 \mathrm{~mL}$ of NS) and call HO

- Check FSBS in 15 minutes.

- Repeat IV 50\% dextrose until FSBS is $>70 \mathrm{mg} / \mathrm{dL}$.

- Once FSBS is $>70 \mathrm{mg} / \mathrm{dL}$, recheck FSBS in 1 hour; if it is $<70 \mathrm{mg} / \mathrm{dL}$, repeat above cycle and call $\mathrm{HO}$.

Patient NPO or unconscious and no IV access available

- Administer glucagon 1 mg IM.

- Turn patient on side (to avoid broncho-aspiration) and call HO.

- Check FSBS in 15 minutes.

- If still $<70 \mathrm{mg} / \mathrm{dL}$, start IV line and follow protocol for an unconscious patient with IV access available.

\section{Special considerations}

Steroid use. No clinical trials have been conducted to define a quantitative approach to managing hyperglycemia induced by steroids (in those patients without previous diabetes) or to understand adjustments in insulin dose for diabetic patients who will undergo treatment with steroids.

Empirically, we recommend the use of NPH insulin and to adjust the dose calculation $20 \%$ higher for low-dose prednisone (10-20 mg/day), $30 \%$ higher for medium-dose prednisone (21-40 mg/day), and 50\% higher for high-dose prednisone ( $>41 \mathrm{mg} /$ day). We do not recommend the use of glargine insulin in this setting since the half-life of prednisone is less than 24 hours; hence, the risk of hypoglycemia is high when using very long-acting insulin. Also, empirically, we make the recommendation to maximize the NPH AM dose and minimize the PM dose, possibly dividing the calculated dose into three quarters AM, one quarter PM. We also caution that this approach would not be appropriate when a patient is using other steroids (prednisolone, methylprednisolone, and dexamethasone) because the half-life of these steroids exceeds 24 hours, and in such cases glargine insulin may be suitable.

Enteral and parenteral feeding. Frequently patients need enteral or parenteral feeding. The former may be given as continuous or discontinuous infusion; hence, in this particular setting, a specific insulin regimen must be customized in close collaboration with the dietician. For example, for those patients who are chronically fed enterally and for whom a system of bolus has been established, the use of a basal insulin may be warranted. However, for the patient who is being fed nocturnally only, we would probably choose NPH as the insulin regimen. Good success has been found in some hospitals with the use of premixed insulin preparations when enteral feeding is continuous for 24 hours. Patients fed parenterally may receive their basal and alimentary insulin as an addition to the nutrition bag, complemented with correction insulin administered subcutaneously.

\section{CONCLUSIONS}

In conclusion, it is the responsibility of hospitalists to make a conscious effort to manage hyperglycemia in patients who are previously diabetic or become hyperglycemic during hospitalization in order to improve their clinical outcome. Hospitals need to realize that this task is far from being the "lone" duty of physicians; hence, systems for hyperglycemia management that engage multidisciplinary teams must be established.

Although the ideal way to do so in the critical care setting is continuous intensive insulin infusion therapy, this may not always be practical. In such cases, basal and alimentary insulin with appropriate insulin sliding scales should be used. Using the sliding scales alone should be strongly discouraged, as they tend to only troubleshoot a situation and allow the damage caused to the patients on a molecular level to be camouflaged in objective ways. Appropriate attention should be paid to the risk of developing hypoglycemia as a sequela of overzealous correction of hyperglycemia. This leaves us with the therapeutically desirable band of the glycemic spectrum. However, this band is wide enough to make it possible to achieve better performance. Although the target glycemic range definitively needs to be determined, it is reasonable to have $80-110 \mathrm{mg} / \mathrm{dL}$ as the target range for critically ill patients, as generally agreed by both the ACE and ADA, and a glycemic range of preprandial glucose between 90 and $130 \mathrm{mg} / \mathrm{dL}$ for ambulatory patients. The maximal blood glucose should not exceed 180 $\mathrm{mg} / \mathrm{dL}$. Having realized the adverse impact on patients of uncontrolled hyperglycemia, at the next morning report, it is appropriate for the nurse to say, "Mr. Smith's finger-stick glucoses are better controlled now, and he required only 2 units of additional insulin coverage yesterday." If we still hear high glucose numbers and keep fixing this problem with sliding-scale insulin alone, we are not doing a good job. 
Address for correspondence and reprint requests: Deepak Asudani, MD, 1400 State St., Springfield, Massachusetts 01109; E-mail: deepak.asudani@bhs.org.

Received 17 0ctober 2006; revision received 15 December 2006; accepted 17 December 2006

\section{REFERENCES}

1. Umpierrez GE, Isaacs SD, Bazargan N, You X, Thaler LM, Kitabchi AE. Hyperglycemia: an independent marker of inhospital mortality in patients with undiagnosed diabetes. J Clin Endocrinol Metab. 2002;87:978-982.

2. Capes S, Hunt D, Malmberg K, Gerstein H. Stress hyperglycaemia and increased risk of death after myocardial infarction in patients with and without diabetes: a systematic overview. Lancet. 2000;355:773-778.

3. Van den Bergh G. Insulin therapy for the critically ill patient. Clin Cornerstone. 2003;5(2):56-63.

4. Pomposelli J, Baxter J, Babineau T, et al. Early postoperative glucose control predicts nosocomial infection rate in diabetic patients. J Parenter Enter Nutr. 1998;22:77-81.

5. Ling PR, Bistrian BR, Mendez B, Istfan NW. Metabolism. 1994;43:279-284.

6. Malmberg K, Rydén L, Efendic S, et al. Randomized trial of insulin-glucose infusion followed by subcutaneous insulin treatment in diabetic patients with acute myocardial infarction (DIGAMI study): effects on mortality at 1 year. $J \mathrm{Am}$ Coll Cardiol. 1995;26:57-65.

7. Van den Berghe G, Wouters P, Weekers F, et al. Intensive insulin therapy in critically ill patients. $N$ Engl J Med. 2001; 345:1359-1367.

8. Van den Bergh G, Wilmer A, Hermans G, et al. Intensive insulin therapy in the medical ICU. $N$ Engl J Med. 2006;354: 449-461

9. Pittas AG, Siegel RD, Lau J. Insulin therapy for critically ill hospitalized patients: a meta-analysis of randomized controlled trials. Arch Intern Med. 2004;164:2005-2011.

10. Krinsley J. Perioperative glucose control. Curr Opin Anaesthesiol. 2006;19(2):111-116.

11. Golden S, Peart-Vigilance C, Kao W, Brancati F: Perioperative glycemic control and the risk of infectious complications in a cohort of adults with diabetes. Diabetes Care. 1999;22:1408-1414.

12. Baker EH, Janaway $\mathrm{CH}$, Philips BJ, et al. Hyperglycaemia is associated with poor outcomes in patients admitted to hospital with acute exacerbations of chronic obstructive pulmonary disease. Thorax. 2006;61:284-289.

13. Clement S, Braithwaite SS, Magee MF, et al.; American Diabetes Association Diabetes in Hospitals Writing Committee. Management of diabetes and hyperglycemia in hospitals. Diabetes Care. 2004;27:553-591. Errata in: Diabetes Care. 2004;27:856. Hirsh, Irl B [corrected to Hirsch, Irl B]; dosage error in text; and Diabetes Care. 2004;27:1255

14. Garber AJ, Moghissi ES, Bransome ED Jr, et al. American College of Endocrinology position statement on inpatient diabetes and metabolic control. Endocr Pract. 2004;10(1):7782.

15. Inzucchi SE, Rosenstock J. Counterpoint: inpatient glucose management: a premature call to arms? Diabetes Care. 2005; 28:976-979

16. Joint Commission on Accreditation of Health Organizations Web site. Avail at: http://www.jointcommission.org/NewsRoom/NewsReleases/jc_nr_072006.htm. Accessed September 29, 2006. 